\title{
Psicologia Pediátrica: A Atenção à Criança e ao Adolescente com Problemas de Saúde
}

Pediatric Psychology:

The care of children and adolescents with health problems

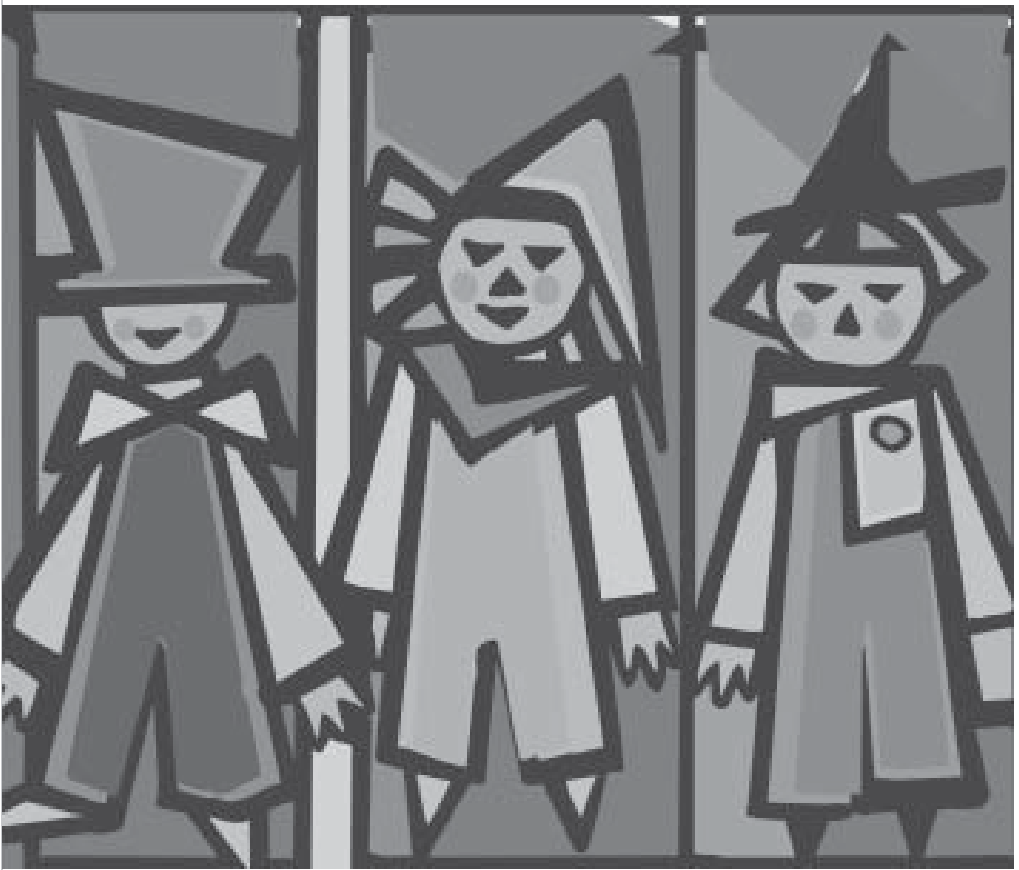




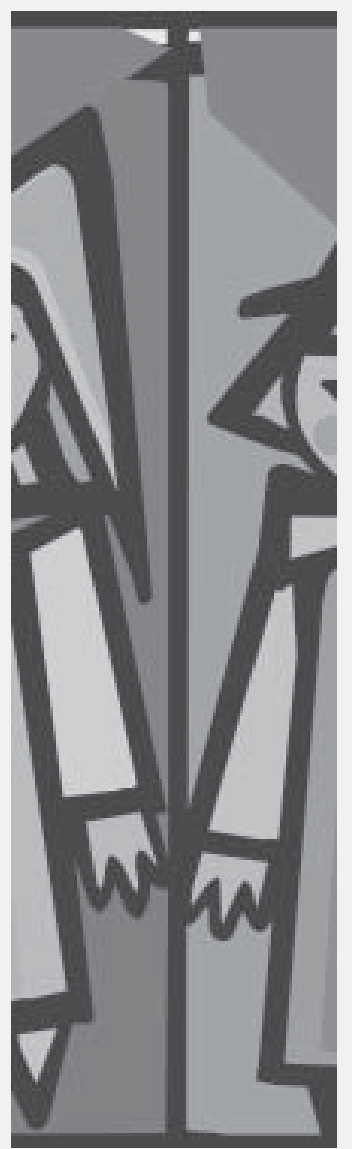

Resumo: O presente trabalho examina algumas questões referentes ao modelo psicossocial, à Psicologia da saúde e à Psicologia pediátrica. A Psicologia pediátrica é o campo que aplica os conhecimentos da Psicologia da saúde às crianças e adolescentes com problemas de saúde e tem como objetivo prevenir, compreender e tratar os problemas psicológicos causados pelos transtornos físicos. A autora discute as origens da Psicologia pediátrica, os conhecimentos básicos para trabalhar nesse campo e questões referentes à atuação prática e à pesquisa com crianças e adolescentes com problemas de saúde e suas famílias.

Palavras-chave: Psicologia pediátrica, Psicologia da saúde, modelo biopsicossocial, formação profissional.

Abstract: The present work examines some questions concerning the biopsychosocial model, Health Psychology and Pediatric Psychology. Pediatric Psychology is Health Psychology applied to children and adolescents with health problems and it aims to prevent, understand and treat psychological problems caused by physical conditions. The author discusses the origins of Pediatric Psychology, the background to operate in this field, questions regarding practical work and research with children and adolescents with health problems and their families.

Key words: Pediatric Psychology, Health Psychology, biopsychosocial model, professional studies.

Os avanços nos cuidados médicos levaram à melhoria na sobrevivência de crianças com doenças que antes eram fatais e agora se tornaram crônicas (Eiser, 1996; Eiser, 1985). As conseqüências psicológicas da doença e do tratamento podem ser várias, a curto e a longo prazo, para a criança e a família. Isso exige, da Psicologia, cada vez mais conhecimentos para atender e tratar crianças e adolescentes que convivem diariamente com doenças graves ou são sobreviventes de problemas que, até poucos anos atrás, levavam à morte. Por isso, a Psicologia pediátrica aplica os conhecimentos da Psicologia da saúde ao cuidado da criança e do adolescente doente, bem como às suas famílias (APA, 2006).

O presente artigo visa a apresentar a Psicologia pediátrica, configurando-a como uma área de conhecimento e de atuação do psicólogo da saúde. Na primeira parte do trabalho, examinam-se as mudanças geradas com a mudança de paradigma do modelo biomédico ao modelo biopsicossocial da saúde. Em seguida, revisam-se aspectos relacionados à Psicologia da saúde como os pilares da Psicologia pediátrica. Por fim, explica-se a Psicologia pediátrica como um campo de trabalho que está em expansão e que tem diversas aplicações práticas.

\section{Do modelo biomédico ao modelo biopsicossocial da saúde}

O modelo que tem sido dominante no mundo ocidental para tratar da saúde e da doença é o biomédico, que pressupõe que os responsáveis 
pelos problemas de saúde sejam os agentes etiológicos específicos que alteram as estruturas e/ou funções do corpo humano (Bowling, 2002). Essa visão, baseada na filosofia cartesiana, entende o corpo como uma máquina: se uma parte funciona mal, esta pode ser reparada ou trocada. Apesar de as doenças poderem causar transtornos psicológicos, os fatores psicológicos não são capazes de causálos, de acordo com esse posicionamento. Mente e corpo são duas entidades consideradas separadamente.

Em contrapartida, o modelo biopsicossocial "A definição organicista da saúde está sendo superada por uma dimensão que é também psicológica e social, pois essa é a única via para o desenvolvimento de políticas de prevenção e promoção da saúde."

González-Rey compreende a saúde como fruto de uma combinação de vários fatores, incluindo características biológicas (por exemplo, predisposição genética), fatores de comportamento (ex: estilo de vida) e condições sociais (ex: influências culturais) (APA, 2006; Bowling, 2002; Suls \& Rothman, 2004; WHO, 2004). Desse modo, a saúde e a doença não são opostas, e sim, compreendidas como um continuum. De acordo com esse modelo, o atual conceito de saúde proposto pela Organização Mundial da Saúde inclui o bemestar físico, mental e social, e valoriza a percepção pessoal e subjetiva do indivíduo como um fator fundamental a ser considerado (WHO, 2004).

No que se refere à saúde, portanto, existe a mudança de enfoque do modelo biomédico ao modelo biopsicossocial, que vai da fisiologia individual à cultura, das intervenções individuais às políticas públicas, da prevenção primária aos cuidados paliativos (Smith, Kendall \& Keefe, 2002). A definição organicista da saúde está sendo superada por uma dimensão que é também psicológica e social, pois essa é a única via para o desenvolvimento de políticas de prevenção e promoção da saúde (González-Rey, 1997). O organismo humano é uma organização complexa e integrada, e existem diferentes maneiras de possibilitar um desenvolvimento saudável. A dimensão subjetiva, certamente, é um aspecto muito importante a ser avaliado. No entanto, para
López-Roig, Pastor e Neipp (2003), o modelo biomédico segue sendo o paradigma dominante na área da saúde, apesar das mudanças ocorridas nos últimos anos, e, com ele, os avanços biomédicos são vistos como os únicos responsáveis pelas mudanças em saúde, para muitos profissionais.

\section{Psicologia da saúde}

A Psicologia da saúde é parte da mudança de paradigma do modelo biomédico de atenção à saúde para o modelo biopsicossocial. É uma disciplina ampla, que surgiu da necessidade de novos parâmetros em saúde e que manifesta as transformações pelas quais passa a sociedade (Angerami-Camon, 2000; Castro \& Bornholdt, 2004; Remor, 1999; Smith \& Suls, 2004). Johnston e Kennedy (1998) a definem como o estudo dos processos psicológicos e comportamentais na saúde, enfermidade e cuidados com a saúde. Tem como foco os problemas físicos de saúde e traz, como resultado, novas perspectivas teóricas, modelos de medidas e delineamento de intervenções. Inicialmente, teorias e métodos de outras áreas básicas da Psicologia foram utilizados para tratar o tema da saúde física, e avaliações e intervenções de outras áreas foram aplicadas no contexto da saúde (Smith \& Suls, 2004). No entanto, com o aumento expressivo no número de pesquisas e com o crescimento dos serviços clínicos de saúde, esse campo vem se desenvolvendo muito nos últimos anos. O grande avanço da ciência biomédica e da Psicologia deu novas e melhores oportunidades para situar o trabalho em Psicologia da saúde. Devido às mudanças que essa disciplina está passando e a seus campos de confluência com a Medicina, saúde pública e fatores econômicos associados aos serviços de saúde, são necessários recursos adicionais para pesquisas e educação para a saúde.

No Brasil, a Psicologia da saúde se confunde com a chamada Psicologia hospitalar (Castro \& Bornholdt, 2004; Chiattone, 2000; 
Yanamoto, Trindade \& Oliveira, 2002). No entanto, a Psicologia da saúde é muito mais ampla, pois abarca intervenções no âmbito primário, secundário e terciário, que podem ser realizadas não somente no hospital mas também na comunidade, em escolas, clínicas, etc. A Psicologia hospitalar seria, então, a atuação do psicólogo que utilizasse os conhecimentos da Psicologia da saúde dentro do hospital.

A provocativa premissa inicial de que as experiências psicológicas e as condutas sociais das pessoas estão relacionadas, de maneira recíproca, com os processos biológicos permitiram avanços importantes no campo da Psicologia da saúde nos últimos vinte e cinco anos, estimulando inovações no seu delineamento e a implementação de programas de promoção para a saúde (Suls \& Rothman, 2004). Como ponto de partida, o modelo biopsicossocial tem tido grande êxito, e está dando oportunidade, a muitos psicólogos da saúde, de abordarem o funcionamento humano a partir de vários níveis e sistemas. Esses avanços no campo estão sendo complementados pelo aumento do número de psicólogos da saúde em universidades e escolas médicas e pelo aumento do apoio a investigações psicológicas sobre condutas relacionadas à saúde.

Os psicólogos da saúde estão realizando importantes avanços na identificação das preocupações e necessidades primárias associadas às doenças crônicas, problemas psicofisiológicos, sintomas médicos persistentes e no desenvolvimento de avaliações válidas para medir resultados clínicos em várias culturas (Nicassio, Meyerowitz \& Kerns, 2004). Os psicólogos também estão obtendo sucesso em adaptar tratamentos psicológicos para sua aplicação na saúde, como intervenções destinadas a melhorar comportamentos de educação para a saúde, redução de estresse, bloqueio emocional e auto-regulação. Em algumas dessas áreas, são examinadas a importância do gênero, idade, diversidade racial e étnica, etc., e as intervenções variam de uma abordagem genérica a uma abordagem específica. Fatores de influência na doença, como sua duração, ambiente cultural, fatores pessoais, etc., são importantes, e devem ser considerados como moderadores no desenvolvimento dessas intervenções.

Existe uma necessidade geral de aplicar, de maneira mais eficiente, a pesquisa em Psicologia da saúde à prática clínica e às políticas de saúde, e vice-versa (Remor, 1999; Suls \& Rothman, 2004). É freqüente encontrar estudos importantes com possibilidades de aplicações práticas que não são implementadas. Por exemplo, identificam-se fatores de risco para o uso do cigarro em adolescentes, mas não se realizam estudos que avaliem intervenções nos hábitos de saúde dessa população. Do mesmo modo, alguns pesquisadores não sabem como seus resultados e recomendações podem ter efeitos práticos efetivos. Esse tema é curioso, pois a Psicologia da saúde amadureceu o suficiente para apoiar e gerar avaliações e intervenções que proporcionem aos profissionais um trabalho eficiente que compete com a abordagem biomédica tradicional. De acordo com Nicassio, Meyerowitz e Kerns (2004), os obstáculos que interferem na aplicação prática dos conhecimentos obtidos nas investigações nesse campo são basicamente três: falta de conhecimento por parte dos investigadores sobre a aplicabilidade e a relevância clínica das intervenções que traçam, a relutância dos profissionais em aceitar o valor efetivo de intervenções baseadas em teorias ou falta de conhecimentos para aplicá-las e as barreiras institucionais diversas, como a falta de pessoal apropriado para levar adiante os projetos, as limitações financeiras, etc. Segundo Johnston e Kennedy (1998), deve haver um treinamento específico dos pesquisadores em relação aos modelos e serviços de atenção ao paciente, assim como os psicólogos clínicos devem adquirir conhecimentos e ter especialização numa prática que vá além do trabalho clínico tradicional individual.
A provocativa premissa inicial de que as experiências psicológicas e as condutas sociais das pessoas estão relacionadas, de maneira recíproca, com os processos biológicos permitiram avanços importantes no campo da Psicologia da saúde nos últimos vinte e cinco anos, estimulando inovações no seu delineamento e a implementação de programas de promoção para a saúde

Suls \& Rothman 
Os estudos e a aplicabilidade da Psicologia da saúde podem ser considerados principalmente em três âmbitos: 1) manejo da doença crônica ou da doença de longo tratamento (câncer, diabete, etc.); 2) tratamento dos problemas psicofisiológicos (como hipertensão, insônia, dor de cabeça causada pela tensão, etc.), e 3) cuidado com os pacientes com sintomas refratários (como a dor), com comorbidades psicológicas (como ansiedade, depressão), e a pacientes que devem ser submetidos a uma cirurgia ou a outros procedimentos médicos estressantes (Nicassio, Meyerowitz \& Kerns, 2004). Justamente para ser capaz de realizar esse tipo de trabalho, a formação do psicólogo da saúde deve contemplar, fundamentalmente, as bases biológicas, sociais e psicológicas da saúde e da doença, a avaliação e a intervenção em saúde, as políticas de saúde e organização, o trabalho interdisciplinar, os temas legais e éticos e conhecimentos de metodologia de pesquisa em saúde (Besteiro \& Barreto, 2003).

O objetivo primordial da pesquisa em saúde é compreender como as pessoas adoecem, suas percepções, comportamentos e experiências relacionadas à saúde e aos efeitos da doença, e sua experiência com os serviços de saúde, incluindo o afrontamento e o manejo de estratégias, etc. (Bowling, 2002). No ambiente hospitalar, Ulla e Remor (2003) destacam algumas razões pelas quais o psicólogo da saúde deve realizar pesquisas sistemáticas: para melhorar a atenção aos doentes e certificar-se de que as intervenções realizadas sejam as mais eficazes, para diminuir os custos em recursos materiais e humanos e para aumentar os conhecimentos sobre o comportamento humano em situação de saúde e doença. Para que os estudos realizados correspondam ao modelo biopsicossocial, é necessário, de acordo com Suls e Rothman (2004), que quatro domínios principais sejam contemplados - aspectos biológicos, psicológicos, sociais e culturais. Uma revisão dos estudos nesse campo, realizada pelos autores, constatou que os psicólogos se esforçam para atuar nesse modelo, mas as pesquisas ainda enfatizam somente os fatores psicológicos e sociais ou os fatores psicológicos e biológicos.

O impacto e o significado futuro da Psicologia da saúde dependerá da efetividade do trabalho dos pesquisadores, dos clínicos, das políticas públicas (Nicassio, Meyerowitz \& Kerns, 2004) e da habilidade para integrar a avaliação e o manejo da saúde física e mental nos cuidados médicos (Smith, Kendall \& Keefe, 2002). Devido à natureza desse campo, supõe-se que a abordagem interdisciplinar seja fundamental para o desenvolvimento de intervenções que devem ser derivadas de evidências empíricas, sensíveis às diferenças de gênero, status socioeconômico e educacional, dentre outras variáveis, em diversos contextos e ambientes.

\section{Psicologia pediátrica}

O termo Psicologia pediátrica (Pediatric Psychology) surgiu, pela primeira vez, em 1968, quando cientistas e profissionais interessados no cuidado da saúde de crianças, adolescentes e suas famílias, ao reconhecerem a necessidade de avaliar e intervir junto esses indivíduos, se reuniram para formar a divisão 54 da American Psychological Association (APA, 2006). Desde então, essa área tem crescido e se ampliado. A definição atual de Psicologia pediátrica se refere à aplicação dos conhecimentos da Psicologia da saúde para a criança, adolescente e suas famílias. Portanto, ela pode ser considerada uma subárea da Psicologia da saúde, que abrange o atendimento clínico, a pesquisa e o ensino (Miyasaki \& cols., 2002).

A divisão 54 da APA tem contribuído ativamente para o avanço da área, e publica o periódico Journal of Pediatric Psychology, de grande reconhecimento internacional. Segundo essa sociedade (APA, 2006), os profissionais da área atuam, de maneira multidisciplinar, em hospitais pediátricos, clínicas infantis e centros de saúde, assim como na clínica tradicional ou área acadêmica. No Brasil, não existe uma 
sociedade formada por psicólogos que trabalham na área; no entanto, a Associação Nacional de Pesquisa e Pós-graduação em Psicologia (ANPEPP, 2006) tem um grupo de trabalho sobre Psicologia pediátrica que tem contribuído para o avanço desse campo de estudo no País.

A formação do psicólogo que trabalha em Psicologia pediátrica deve contemplar vários domínios (Spirito, Brown, D'Angelo \& cols., 2003): ciclo evolutivo vital, psicopatologia do desenvolvimento, avaliação da criança, do adolescente e de sua família, estratégias de intervenção e métodos de investigação e sistemas de avaliação, o papel das várias disciplinas relacionadas com a atenção à infância, prevenção, apoio familiar e promoção da saúde, aspectos sociais que afetam a criança, o adolescente e sua família e processo da doença e manejo médico, entre outras. Assim, as atividades que pode realizar um psicólogo que atua na área são bastante amplas, incluindo a atuação em equipes interdisciplinares para acompanhar crianças doentes, a preparação psicológica para cirurgia e outros procedimentos médicos, a avaliação psicológica, a realização de grupos de orientação a pais de crianças com problemas específicos de saúde, grupos de apoio, e a atuação em atividades de extensão à comunidade. (Maloney, Clay \& Robinson, 2005; Miyasaki \& cols., 2002). Segundo Sierra e Bermúdez (2005), a formação em Psicologia pediátrica na América Latina é deficiente, e não está presente nos currículos da grande maioria das universidades.

\section{A doença crônica na infância e a Psicologia pediátrica}

A doença crônica infantil pode ser vista como um estressor complexo, em que se somam as hospitalizações, as vivências de dor, as explorações médicas, os remédios, as restrições nas atividades diárias, os temores, etc. (Trianes, 2002). Seu impacto sobre a criança dependerá da duração, sintomatologia, gravidade, visibilidade da doença, tipos de intervenções médicas que requer, características da criança e das relações familiares. A hospitalização pode ter conseqüências imediatas para algumas crianças, como o aparecimento de comportamentos agressivos, pesadelos, mudanças na conduta, medo de separar-se de seus pais, etc. É importante examinar se os comportamentos observados nas crianças doentes são primários ou secundários à enfermidade, para melhor compreender suas reações (Lewis \& Kellet, 2004).

O conceito de doença, para crianças e adolescentes, passa por um processo evolutivo que está mais diretamente relacionado ao seu nível de desenvolvimento cognitivo e à idade em que passou pela experiência (RodríguezMarín \& cols., 2003). No entanto, o estudo realizado por Torres (2002) sobre a relação entre nível cognitivo e conceito de doença e morte com crianças doentes crônicas e crianças saudáveis mostrou resultados mais complexos. Foi encontrada uma defasagem cognitiva nas crianças doentes com relação às crianças saudáveis e um impacto desestruturante da doença na aquisição do conceito de morte no período pré-operacional. Por outro lado, quando as crianças atingem o nível das operações concretas, a situação se modifica, e os dados revelaram que a doença funcionou como um fator de maturação do conceito de morte. Assim, a compreensão que a criança tem de sua doença é um dos fatores importantes para a sua adaptação ou não à situação (Trianes, 2002). A criança pode entender a doença como uma mudança, uma perda irreparável e irreversível, um inimigo, um castigo por alguma falta cometida, uma oportunidade para não ir à escola, uma estratégia para atrair a atenção ou uma oportunidade para o crescimento e o desenvolvimento pessoal, entre outros.

Hospitalizações, procedimentos médicos e cirurgias são eventos estressantes para crianças e adolescentes em todas as idades (Aley, 
2002). Essas experiências são geralmente traumáticas, e trazem, como resultado, um sentimento de insegurança, de falta de ajuda, de medo intenso e de ansiedade. A repetição de hospitalizações e experiências médicas estressantes pode prejudicar os desenvolvimentos cognitivo, emocional, físico e social da criança. É essencial que os profissionais de saúde saibam reconhecer as situações potencialmente estressantes para introduzir intervenções apropriadas e facilitar a utilização do potencial de crescimento de cada criança. Ao mesmo tempo, as hospitalizações provocam situações que permitem, ao psicólogo, observar o efeito das separações da criança com seus progenitores (Bowlby, 1983). Os efeitos disso dependerão muito do tempo da separação, com quem e em que lugar fica a criança, sua idade, experiências prévias, etc. Para Bowlby (1983), o desapego frente à separação deve ser entendido como um processo defensivo por parte da criança.

O recurso interpessoal mais importante que as crianças podem ter frente às situações de doença, dor e hospitalização é o apego seguro aos seus cuidadores (Trianes, 2002). São eles que vão ajudá-la a enfrentar as dificuldades e a modificar seu estilo de vida, especialmente quando a criança é pequena. Com o passar do tempo, as crianças assumem, pouco a pouco, sua própria capacidade de lidar com a situação.

O ajustamento à doença crônica tem sido relacionado com o desenvolvimento de estratégias de enfrentamento e de defesas específicas por parte da criança (Eiser, 1985). As habilidades de enfrentamento infantil são diferentes das habilidades dos adultos, especialmente porque as crianças têm um repertório limitado de estratégias devido ao seu estágio de desenvolvimento e a sua pouca experiência (Trianes, 2002). Eiser (1985) caracteriza as estratégias em cinco tipos: 1 ) funções cognitivas de memória, linguagem e pensamento: fazem com que a criança esteja apta para aceitar as limitações impostas pela doença, assumir a responsabilidade de seu próprio cuidado e cooperar com o manejo médico; 2) atividades compensatórias físicas e intelectuais: desenvolvimento de novas áreas de interesse e de funcionamento adaptativo; 3) controle apropriado das emoções: a criança pode aprender a expressar a frustração e a raiva associada à doença de maneira socialmente aceitável e em ocasiões apropriadas; 4) controle do isolamento: é apropriado que a criança tenha atividades sociais, e 5) estratégias defensivas para o manejo da doença: o grau de negação do problema associado à doença pode ser útil em alguns casos, quando a criança tem que passar por uma situação extremamente ansiogênica. Trianes (2002) afirma que, frente aos estressores médicos, as crianças costumam utilizar, com mais freqüência, as estratégias de acomodação à situação, rebelando-se pouco frente à situação e aceitando sua condição.

O jogo é a maneira pela qual a criança expressa sua compreensão do mundo, como se desenvolve e aprende, e elabora, ao mesmo tempo, seu luto e experiência pessoal. O jogo pode favorecer o desenvolvimento da criança em situações de doença e hospitalização e ter um efeito terapêutico (Aley, 2002; Quiles, Ortigosa \& Méndez, 2003). No entanto, o jogo terapêutico é diferente de outros tipos de jogos, por ser facilitado por um terapeuta treinado e especializado, que trata de temas relacionados aos medos e ao bem-estar psicossocial do paciente (Aley, 2002). Esse profissional pode utilizar as brincadeiras de médico para recriar as percepções das crianças sobre suas experiências, e dá à criança a oportunidade de se comunicar com o terapeuta. Do mesmo modo, o trabalho com desenhos permite à criança ilustrar seus medos, preocupações e fantasias. Os centros hospitalares infantis deveriam, também, preocupar-se em proporcionar atividades lúdicas e aulas escolares para manter as crianças centradas em interesses de aprendizagem e desenvolvimento pessoal, a fim de que elas não estejam, todo o tempo, preocupadas com a doença (Trianes, 2002). 
Com relação aos estudos feitos com crianças e adolescentes doentes, Eiser (1996) ressalta que devemos nos preocupar com a adoção de modelos teóricos apropriados para essa população. Segundo essa autora, nem sempre os delineamentos de pesquisa tradicionais de comparação de crianças doentes com um grupo controle são os mais apropriados. É importante observar as diferenças existentes dentro da mesma amostra de crianças doentes para compreender as particularidades existentes entre crianças adaptadas e com problemas psicológicos. Além disso, os problemas, dificuldades e preocupações das crianças doentes não deveriam ser o único enfoque das pesquisas com essa população, pois isso vai contra a idéia de que alguns pacientes lidam de maneira satisfatória com sua doença. Ao mesmo tempo, existem problemas metodológicos importantes na realização de estudos com crianças enfermas, especialmente porque são necessárias grandes amostras para que se verifiquem diferenças entre grupos e subgrupos, o que dificilmente é possível quando se trabalha com esse tipo de sujeitos. A solução freqüentemente encontrada pelos pesquisadores é a análise de pequenos grupos de participantes, que resulta numa avaliação não compreensiva das diferentes variáveis investigadas e que não explica os processos psicológicos relacionados à doença infantil.

Em muitas pesquisas com crianças com problemas de saúde, comparam-se dois ou mais grupos (Lewis \& Kellet, 2004). Em geral, a idéia é verificar como um tipo de doença afeta o desenvolvimento, mas a dificuldade está em selecionar grupos de comparação adequados. Outra questão importante relaciona-se à adequação ou não de agrupar crianças com diferentes problemas num mesmo grupo, o que supõe que cada indivíduo de um grupo compartilhe algumas características. Esse problema é especialmente importante se pensarmos que a natureza dos problemas dos indivíduos pode ser diferente, e que, além disso, o assunto se torna ainda mais complicado nos casos de pessoas com múltiplos e/ou sérios transtornos.

Kellet e Ding (2004) apontam outro aspecto fundamental da investigação com crianças, que se refere à fonte de dados. Para esses autores, as próprias crianças são as pessoas mais indicadas para fornecer dados sobre si mesmas e sobre o que as preocupa. Para isso, um bom rapport é fundamental para que a criança não se sinta intimidada pelo investigador. Com relação aos procedimentos, as entrevistas gravadas, os grupos de discussão e as observações são vantajosos no trabalho com essa população. Por outro lado, os questionários ao estilo de adultos podem ser problemáticos se as questões forem complexas e porque essa é uma tarefa de pouco interesse para a criança.

Há vários usos e aplicações dos estudos com a população pediátrica. McKechnie e Hobbs (2004) destacam algumas: 1) uso instrumental, em que os resultados dos estudos vão diretamente à prática; 2) uso conceitual, em que as evidências influenciam idéias e mostram novos caminhos para pensar temas importantes; 3) apoio, em que as evidências são usadas para persuadir outros, pensandose sobre a ação, e 4) influência ampla, em que a pesquisa exerce uma influência em toda a comunidade e pode influenciar paradigmas e políticas de saúde/educação. Com relação aos programas e intervenções com a população pediátrica, teoricamente, a melhor maneira custo-benefício de prevenir problemas adicionais à doença é intervir o mais cedo possível e no momento evolutivo mais apropriado (Zeiner, Bendell \& Walker, 1985). Os psicólogos da saúde deveriam promover mais esforços em investigações com esses pacientes.

\section{Considerações finais}

No presente trabalho procurei sintetizar o que é a Psicologia pediátrica, considerando-a uma área que está incluída na Psicologia da saúde. 
Sua tarefa fundamental é ajudar a melhorar o bem-estar e a qualidade de vida do paciente e de sua família, considerando a saúde um conceito multidimensional que abarca os aspectos físicos, psicológicos e sociais.

A Psicologia pediátrica é um campo que vem se consolidando internacionalmente, e também no Brasil. Embora provavelmente exista um grande número de psicólogos trabalhando nesse ramo, a definição do que seria um trabalho em Psicologia pediátrica ainda é escassa. Não existem associações e/ ou sociedades de profissionais que trabalhem especificamente nessa área, mas apenas grupos de trabalho, como no caso da ANPEPP - Associação Nacional de Pesquisa e Pósgraduação em Psicologia. A formação de tais associações ajudaria no avanço do campo, tanto em investigação como na sua aplicabilidade.

As implicações psicológicas relacionadas à saúde da criança e do adolescente podem ser tão variadas como tão diversos também são os problemas de saúde que eles podem apresentar. As conseqüências emocionais dos problemas de saúde da criança para a sua família direta também são inúmeras. Por isso, o psicólogo pediátrico deve estar treinado para lidar com situações muito complexas, que envolvam o bem-estar psicológico não só da criança/adolescente mas também de todos os que o rodeiam.

Ao mesmo tempo em que é importante uma atividade clínica efetiva e eficaz em Psicologia pediátrica, são necessárias mais pesquisas que tratem da saúde da criança/adolescente brasileiro a fim de dar base a essas intervenções. A grande maioria da literatura científica nessa área vem de estudos realizados em países desenvolvidos, e sabemos que nem sempre os resultados encontrados se encaixam ao nosso contexto. Além disso, esses estudos utilizam diferentes critérios e medidas para investigar os aspectos psicossociais da saúde da criança, geralmente utilizando fontes de informação de terceiros (pais, professores, etc.), já que pouca informação é obtida através da criança.

Os avanços teóricos, metodológicos e clínicos para lidar com a saúde de crianças e adolescentes nos últimos anos são inegáveis, mas ainda temos muito trabalho pela frente. São necessários estudos empíricos rigorosos que considerem as particularidades da criança doente, sua fase evolutiva, suas condições emocionais, familiares e sociais. Torna-se importante estudar e analisar as implicações dos diversos tipos de problemas de saúde e hábitos de saúde para a criança e adolescente, trabalho que ainda está inacabado.

Frente às situações relacionadas à saúde da criança e do adolescente, é fundamental uma abordagem multiprofissional que envolva também os aspectos psicológicos da criança/ adolescente e suas famílias. É necessário que os profissionais da saúde estejam sensibilizados para os aspectos que transcendem o tratamento médico, pois os resultados do tratamento e as intervenções podem ficar comprometidos se não se consideram esses aspectos.

\section{Elisa Kern de Castro}

Professora adjunta do Programa de Pós-Graduação em Psicologia da Universidade do Vale do Rio dos Sinos. Mestre em Psicologia do Desenvolvimento (UFRGS) e Doutora em Psicologia da Saúde

(Universidad Autónoma de Madrid)

UNISINOS - Av. Unisinos, 950, Bairro Cristo Rei. Centro de Ciências da Saúde. CEP 93022-000.

São Leopoldo, RS. E-mail: elisakerncastro@hotmail.com 
ALEY, K. E. Developmental Approach to Pediatric Transplantation. Progress in Transplantation, v. 12, ㄲo 2, pp. 86-91, 2002.

AMERICAN PSYCHOLOGICAL ASSOCIATION (APA). Official site - Pediatric Psychology http://www.apa.org/divisions/div54/ history.htm (23/01/2006).

ASSOCIAÇÃO NACIONAL DE PESQUISA E PÓS-GRADUAÇÃO EM PSICOLOGIA (ANPEPP). Avanços e Desafios Conceituais e Metodológicos da Pesquisa na Área de Saúde da Criança. Grupo de Trabalho sobre Pesquisa em Psicologia Pediátrica. http:// w w w . a n p e p p.org.br/X S i m pos i o / Pesquisa\%20em\%20psicologia\%20pedi\%C3\%A1trica.doc (10/01/2006).

ANGERAMI-CAMON, V. A. Psicologia da Saúde - um Novo Significado para a Prática Clínica. São Paulo: Pioneira Psicologia, 2000.

BESTEIRO, M. M. \& BARRETO, M. P.La Formación de los Profesionales de la Salud: la Contribución del Psicólogo Hospitalario. In: Remor, E.; Arranz, P.\& Ulla, S. (orgs.). El Psicólogo en el Ámbito Hospitalario. Bilbao: Desclée de Brouwer Biblioteca de Psicologia, 2003, pp. 121-138.

BOWLBY, J. La Pérdida Afectiva: Tristeza y Depresión. Trad. A. Baez. Buenos Aires: Paidós, 1983.

BOWLING, A. Research Methods in Health: Investigating Health and Health Services. Buckingham: Open University Press, 2 ed., 2002.

CASTRO, E. K. \& BORNHOLDT, E. Psicologia da Saúde x Psicologia Hospitalar: Definições e Possibilidades de Inserção Profissional. Psicologia: Ciência e Profissão, Brasília, v. 24, n. 3, pp. 48-57, 2004.

CHIATTONE, H. B. C. A Significação da Psicologia no Contexto Hospitalar. In: Angerami-Camon, V. A. (org). Psicologia da Saúde um Novo Significado para a Prática Clínica. São Paulo: Pioneira Psicologia, 2000, pp. 76-90.

EISER, C. The Psychology of Childhood Illness. New York: SpringerVerlag, 1985

EISER, C. Helping the Child with Chronic Disease: Themes and Directions. Clinical Child Psychology and Psychiatry, Londres:UK, v. 1, n. 4, pp. 551-561, 1996

GONZÁLEZ-REY, F. Psicologia e Saúde: Desafios Atuais. Psicologia, Reflexão e Crítica, Porto Alegre, v. 10, no 2, pp. 275-288, 1997.

JOHNSTON, M. \& KENNEDY, P. Editorial: Special Issue on Clinical Health Psychology in Chronic Conditions. Clinical Psychology and Psychotherapy, West Sussex, v. 5, pp. 59-61, 1998.

KELLET, M. \& DING, S. Middle Childhood. In: Fraser, S.; Lewis, V.; Ding. S.; Kellett, M.; Robinson, C. (orgs.). Doing Research with Children and Young People. London: Sage Publications, 2004, pp. 161-175.

LEWIS, V. \& KELLET, M. Disability. In: Fraser, S.; Lewis, V.; Ding. S.; Kellet, M.; Robinson, C. (orgs.). Doing Research with Children and Young People. London: Sage Publications, 2004, pp. 191-205.

LÓPEZ-ROIG, S.; PASTOR, M. A. \& NEIPP, M. C. Aspectos Psicológicos Asociados a la Hospitalización. In: Remor, E.; Arranz, P. \& Ulla, S. (orgs.). El Psicólogo en el Ámbito Hospitalario. Bilbao: Desclée de Brouwer Biblioteca de Psicologia, 2003, pp. 31-48.

MALONEY, R.; CLAY, D. L. \& ROBINSON, J. Sociocultural Issues in Pediatric Transplantation: a Conceptual Model. Journal of Pediatric Psychology, Philadelphia:USA, v. 30, no 3, pp. 235-246, 2005.

MCKECHNIE, J. \& HOBBS, S. Childhood Studies. In: Fraser, S.; Lewis, V.; Ding. S.; Kellett, M.; Robinson, C. (orgs.). Doing Research with Children and Young People. London: Sage Publications, 2004, pp. 270-285.
MIYAZAKI, M. C. O. S.; DOMINGOS, N. A. M.; VALERIO, N. I.; SANTOS, A. R. \& ROSA, L. T. B. Psicologia da Saúde: Extensão de Serviços à Comunidade, Ensino e Pesquisa. Psicologia USP, v. 13, no 1, pp. 29-53, 2002.

NICASSIO, P.M.; MEYEROWITZ, B. E. \& KERNS, R. D. The Future of Health Psychology. Health Psychology, Lon Angeles, USA, v. 23, no 2, pp. 132-137, 2004.

QUILES, M. J.; ORTIGOSA, J. M. \& MÉNDEZ, F. X. Preparación a la Hospitalización y a la Cirurgía. In: Ortigosa, J. M.; Quiles, M. J.Y Méndez, F.X. (orgs.). Manual de Psicología de la Salud con Niños, Adolescentes y Famílias. Madrid: Pirâmide, 2003, pp. 305-324.

REMOR, E. A. Psicologia da Saúde: Apresentação, Origens e Perspectivas. Revista Psico, Porto Alegre, v. 30, no 1, pp. 205-217, 1999.

RODRÍGUEZ-MARÍN, J. En Busca de un Modelo de Integración del Psicólogo en el Hospital: Pasado, Presente y Futuro del Psicólogo Hospitalario. In: Remor, E.; Arranz, P. \& Ulla, S. (orgs.). El Psicólogo en el Ámbito Hospitalario. Bilbao: Desclée de Brouwer Biblioteca de Psicologia, 2003, pp. 831-863.

SIERRA, J. C. \& BERMÚDEZ, M. P. Hacia el Título Iberoamericano de Psicología: Análisis de los Programas Docentes de las Carreras de Psicología en Iberoamérica. Revista Mexicana de Psicología, Mexico:DF, v. 22, pp. 224-242, 2005.

SMITH, T. W.; KENDALL, P. C. \& KEEFE, F. J. Behavioral Medicine and Clinical Health Psychology: Introduction to the Special Issue, a View from the Decade of Behavior. Journal of Consulting and Clinical Psychology, Miami:USA, v. 70, no 3, pp. 459-462, 2002.

SMITH, T. W. \& SULS, J.. Introduction to the Special Section on the Future of Health Psychology. Health Psychology, Los Angeles:USA, v. 23, no 2, pp. 115-118, 2004.

SPIRITO, A.; BROWN, R. T.; D'ANGELO, E.; DELAMATER, A.; RODRIGUEZ, J. \& SIECEL, L. Society of Pediatric Psychology Task Force Report: Recommendations for the Training of Pediatric Psychologists. Journal of Pediatric Psychology, v. 28, no 2, pp. 85-98, 2003.

SULS, J. \& ROTHMAN, A. Evolution of the Biopsychosocial Model: Prospects and Challenges for Health Psychology. Health Psychology, Los Angeles:USA, v. 23, no 2, pp. 119-125, 2004.

TORRES, W. C. O Conceito de Morte em Crianças Portadoras de Doenças Crônicas. Psicologia: Teoria e Pesquisa, Brasilia, v. 18, no 2, pp. 221-229, 2002.

TRIANES, M. V. Estrés en la Infancia: su Prevención y Tratamiento. Madrid: Narcea Ediciones, 2002.

ULLA, S. \& REMOR, E. La Investigación en el Hospital: Tendiendo Puentes entre la Teoría y la Práctica. In: Remor, E.; Arranz, P. \& Ulla, S. (orgs.). El Psicólogo en el Ámbito Hospitalario. Bilbao: Desclée de Brouwer Biblioteca de Psicologia, 2003, pp. 179-208.

WORLD HEALTH ORGANIZATION - WHO. Official website www.who.int (em 30/09/2004).

YANAMOTO, O. H.; TRINDADE, L. C. B. \& OLIVEIRA, I. F. O Psicólogo em Hospitais no Rio Grande do Norte. Psicologia USP, São Paulo, v. 13, no 1, pp. 217-246, 2002.

ZEINER, A. R.; BENDELL, D. \& WALKER, E. Health Psychology: Treatment and Research Issues. New York: Plenum Press, 1985.
Referências 